\title{
Análise comparativa dos histogramas de dose e volume entre planejamentos tridimensionais conformados e convencionais não conformados na radioterapia do câncer de próstata*
}

\author{
Comparative analysis of dose-volume histograms between 3D conformal and conventional \\ non-conformal radiotherapy plannings for prostate cancer
}

Sílvia Moreira Feitosa ${ }^{1}$, Adelmo José Giordani ${ }^{2}$, Rodrigo Sousa Dias ${ }^{1}$, Helena Regina Comodo Segreto $^{3}$, Roberto Araujo Segreto ${ }^{4}$

Resumo OBJETIVO: Analisar, comparativamente, doses de radiação em volumes alvos e órgãos de risco entre planejamentos conformados e não conformados em pacientes com câncer de próstata. MATERIAIS E MÉTODOS: No presente trabalho foram analisados planejamentos de 40 pacientes portadores de câncer de próstata. Foram realizados planejamentos conformados, não conformados isocêntricos e não conformados utilizando a distância fonte-superfície, simulados para cada caso, para comparação das doses em volumes alvos e órgãos de risco. Para a comparação foram analisados os histogramas de dose e volume para volumes alvos e órgãos de risco. RESULTADOS: As medianas das doses foram significativamente menores no planejamento conformado analisando-se os seguintes volumes no reto: $25 \%, 40 \%$ e $60 \%$. As medianas das doses foram significativamente menores no planejamento conformado analisando-se os seguintes volumes na bexiga: $30 \%$ e $60 \%$. As doses medianas foram significativamente menores no planejamento conformado analisando-se as articulações coxofemorais direita e esquerda. As doses máximas, médias e medianas no volume alvo clínico e no volume alvo planejado foram significativamente maiores no planejamento conformado. CONCLUSÃO: O presente estudo demonstrou que por meio do planejamento conformado em pacientes com câncer de próstata é possível entregar doses maiores no volume alvo e doses menores em órgãos de risco.

Unitermos: Câncer de próstata; Radioterapia; Planejamento conformado; Planejamento não conformado.

Abstract OBJECTIVE: The present study was aimed at comparing conformal and non-conformal radiotherapy plans designed for patients with prostate cancer, by analyzing radiation doses in target volumes and organs at risk. MATERIALS AND METHODS: Radiotherapy plans for $\mathbf{4 0}$ patients with prostate cancer were analyzed. Conformal, conformal isocentric and non-conformal plans utilizing the source-surface distance were simulated for each of the patients for comparison of radiation dose in target volumes and organs at risk. For comparison purposes, dose-volume histograms for target volumes and organs at risk were analyzed. RESULTS: Median doses were significantly lower in the conformal planning, with $25 \%, 40 \%$ and $60 \%$ volumes in the rectum and $30 \%$ and $60 \%$ in the bladder. The median doses were significantly lower in the conformal planning analyzing the right and left coxofemoral joints. Maximum, mean and median doses in the clinical target volume and in the planned target volume were significantly higher in the conformal planning. CONCLUSION: The present study has demonstrated that the conformal radiotherapy planning for prostate cancer allows the delivery of higher doses to the target volume and lower doses to adjacent healthy tissues.

Keywords: Prostate cancer; Radiotherapy; Conformal plan; Non-conformal plan.

Feitosa SM, Giordani AJ, Dias RS, Segreto HRC, Segreto RA. Análise comparativa dos histogramas de dose e volume entre planejamentos tridimensionais conformados e convencionais não conformados na radioterapia do câncer de próstata. Radiol Bras. 2009;42(2):69-74.

* Trabalho realizado na Universidade Federal de São Paulo/Es cola Paulista de Medicina (Unifesp/EPM), São Paulo, SP, Brasil. 1. Mestres, Médicos da Universidade Federal de São Paulo/Escola Paulista de Medicina (Unifesp/EPM), São Paulo, SP, Brasil.

2. Mestre, Físico da Universidade Federal de São Paulo/Escola Paulista de Medicina (Unifesp/EPM), São Paulo, SP, Brasil.

3. Doutora, Médica e Professora da Universidade Federal de São Paulo/Escola Paulista de Medicina (Unifesp/EPM), São Paulo, SP, Brasil.

4. Livre-Docente, Médico e Professor da Universidade Federal de São Paulo/Escola Paulista de Medicina (Unifesp/EPM), São Paulo, SP, Brasil.

\section{INTRODUÇÃO}

A radioterapia externa tornou-se universalmente aceita como modalidade terapêu-

Endereço para correspondência: Dra. Sílvia Moreira Feitosa. Rua Seroa da Mota, 23, Apeadouro. São Luís, MA, Brasil, 65031-630. E-mail: silviamfeitosa@yahoo.com.br

Recebido para publicação em 3/6/2008. Aceito, após revisão, em 15/1/2009. tica adequada para câncer de próstata localizado a partir dos anos 50, com o advento de máquinas de megavoltagem (cobalto 60 e aceleradores lineares), em virtude da menor dose na pele e homogeneidade da radiação na profundidade ${ }^{(\mathbf{1})}$.

A técnica de tratamento convencional ou não conformada utiliza arranjo de feixes de quatro entradas (ântero-posterior, 
póstero-anterior, látero-lateral direito e látero-lateral esquerdo) — também conhecida como técnica em quatro campos ou em box - e pode ser realizada utilizando campos isocêntricos ou campos com distância fonte-superfície fixa, técnica de feixes em dois arcos rotatórios de $120^{\circ}$, combinação de ambas ou técnica de três campos (um campo anterior e dois campos laterais paralelos e opostos direito e esquerdo). O tratamento com técnica rotatória $\left(360^{\circ}\right)$ também pode ser utilizado como reforço de dose (em uma segunda fase), porém, com esta técnica a dose na parede anterior do reto é maior ${ }^{(2,3)}$.

Uma das dificuldades dessas técnicas não conformadas para tratamento do câncer de próstata localizado é a dose incidente nos tecidos normais adjacentes. Nestas técnicas, as doses máximas executáveis estão entre 65 e 70 Gy. Tais doses muitas vezes são insuficientes para erradicar as células clonogênicas tumorais, o que dificulta o controle da doença. Estudos realizados pelo Patterns of Care Study, em 1984, demonstraram que a incidência de toxicidade graus 3 e 4 no reto e na bexiga dobraram (de $3,5 \%$ para $6,9 \%$ ) quando doses acima de 70 Gy eram prescritas com o planejamento não conformado ${ }^{(\mathbf{1})}$.

O desenvolvimento tecnológico, na década de 90 , permitiu a realização do planejamento radioterápico conformado. $\mathrm{O}$ sistema de planejamento em três dimensões varia em alguns aspectos, mas é baseado em um princípio comum: o uso de imagens (tomografia computadorizada ou ressonância magnética) para delimitar a próstata e os órgãos de risco e gerar reconstruções tridimensionais de alta resolução. Com esta técnica, consegue-se definição anatômica precisa dos volumes alvos (próstata e vesículas seminais) e dos tecidos normais adjacentes (reto, bexiga e coxofemoral), permitindo conformação individual dos feixes de radiação aos volumes alvos ${ }^{(4,5)}$.

Devido à apreciação volumétrica do alvo, o plano de tratamento é baseado na habilidade de definir cada subvolume anatomicamente dentro do volume total irradiado e calcular precisamente a dose dada em cada ponto. Este fato possibilita escalonamento da dose de radiação, isto é, altas doses nos volumes alvos e limitação da dose e volume nos tecidos normais adja- centes, dentro dos níveis de tolerância pre$\operatorname{conizados}^{(\mathbf{6})}$.

No Brasil, muitos centros de radioterapia ainda não dispõem de sistema de planejamento para realização de tratamento conformado. É de interesse prático comparar os planejamentos conformados isocêntricos e os planejamentos não conformados.

O objetivo do presente trabalho é realizar análise comparativa entre doses de radiação em volumes alvos e órgãos de risco, em planejamentos conformados tridimensionais, não conformados isocêntricos e não conformados utilizando a distância fonte-superfície, para pacientes portadores de câncer de próstata localizado, submetidos à radioterapia.

\section{MATERIAIS E MÉTODOS}

Neste trabalho foram analisados planejamentos de 40 pacientes portadores de câncer de próstata localizado, atendidos no Setor de Radioterapia da Universidade Federal de São Paulo/Escola Paulista de Medicina (Unifesp/EPM), no período de 2004 a 2005. Foram realizados planejamentos conformados isocêntricos, não conformados isocêntricos e não conformados utilizando a distância fonte-superfície fixa, simulados para cada caso para comparação das doses em volumes alvos e órgãos de risco.

Foram incluídos no estudo pacientes com tumores de próstata em estádio clínico T1 a T2c segundo o American Joint Committee on Cancer (2002), com ausência de metástase a distância e de tratamentos cirúrgicos prévios, submetidos ou não a hormonioterapia neoadjuvante.

\section{Métodos de planejamento}

Pré-planejamento - Os pacientes submeteram-se a simulação prévia no simulador. Os pacientes foram posicionados em decúbito dorsal com as mãos na região anterior do tórax, travesseiro sob a cabeça e apoio de pé. $\mathrm{O}$ isocentro pré-tomografia era marcado com a localização de um campo $10 \times 10 \mathrm{~cm}$, com margem inferior coincidindo com o ramo inferior do ísquio e centro do campo entre os ossos do púbis, no campo anterior; rodando o gantry $90^{\circ}$, este era localizado $1,5 \mathrm{~cm}$ posterior à borda anterior do púbis no campo lateral.
Tomografia de planejamento - $\mathrm{O}$ paciente era orientado a realizar ingestão hídrica de $300 \mathrm{ml}$ e fazer uso de fleet via retal duas horas antes da tomografia de planejamento. Esta era realizada na posição da simulação prévia com cortes de 5 e $5 \mathrm{~mm}$ da região pélvica, desde a transição entre a $4^{\mathrm{a}}$ e $5^{\mathrm{a}}$ vértebras lombares até o terço superior do fêmur, incluindo todos os tecidos moles laterais adjacentes.

Delineação de volumes alvos - Para delineação dos volumes alvos foram delimitados o volume tumoral macroscópico (GTV - gross tumor volume), o volume alvo clínico (CTV - clinical target volume) e o volume alvo planejado (PTV - planning target volume) de cada paciente.

Delineação de órgãos de interesse No contorno dos órgãos de interesse a bexiga foi contornada pela parede externa em sua totalidade, o reto foi contornado pela parede externa da junção retossigmoidea até a margem anal, os fêmures foram contornados pela parede externa do osso e desenhados até o trocanter menor.

Técnicas de planejamento - Com o auxílio do sistema de planejamento computadorizado modelo Eclipse (Varian; Palo Alto, EUA), foram realizados dois planejamentos não conformados e um planejamento conformado para os 40 pacientes, sendo estes realizados em duas fases, para o acelerador linear de $6 \mathrm{MeV}$.

No primeiro planejamento não conformado com distância fonte-superfície fixa foi utilizada, na primeira fase de planejamento, técnica de quatro campos (ânteroposterior, póstero-anterior, látero-lateral direito e látero-lateral esquerdo), usando a distância fonte-superfície fixa de $100 \mathrm{~cm}$, até dose de 5.040 cGy, com margens de 1,5 $\mathrm{cm}$ da próstata e vesículas seminais em todas as direções para pacientes de risco intermediário e alto, e margens de $1,5 \mathrm{~cm}$ da próstata e terço proximal de vesículas seminais em todas as direções para pacientes de baixo risco; as isodoses foram normalizadas (no ponto de máximo) e foi escolhida a isodose de $95 \%$, que englobava o volume alvo. Na segunda fase do mesmo planejamento foi realizada técnica de dois arcos rotatórios isocêntricos até dose de 1.980 cGy, sendo dois arcos rotatórios de $120^{\circ}$ com intervalo central anterior e posterior de $60^{\circ}$ (o primeiro arco englobava de 
30 a $150^{\circ}$ e o segundo arco englobava de 210 a $330^{\circ}$ ), com margens de $1,5 \mathrm{~cm}$ englobando próstata e terço proximal das vesículas seminais. Nas duas fases de tratamento a dose total de prescrição foi de $7.020 \mathrm{cGy}$; as isodoses foram normalizadas (no isocentro) e foi escolhida a isodose de $95 \%$, que englobava o volume alvo.

No segundo planejamento não conformado isocêntrico foi usada, na primeira fase técnica de quatro campos isocêntricos (ântero-posterior, póstero-anterior, látero-lateral direito e látero-lateral esquerdo), dose até $5.040 \mathrm{cGy}$, com margens de $1,5 \mathrm{~cm}$ da próstata e vesículas seminais em todas as direções para pacientes de risco intermediário e alto, e margens de 1,5 cm da próstata e terço proximal das vesículas seminais em todas as direções para pacientes de baixo risco; as isodoses foram normalizadas (no isocentro) e foi escolhida a isodose de $95 \%$, que englobava o volume alvo. Na segunda fase do mesmo planejamento foi utilizada técnica de dois arcos rotatórios isocêntricos até dose de 1.980 cGy, sendo dois arcos rotatórios de $120^{\circ}$ com intervalo central anterior e posterior de $60^{\circ}$ (o primeiro arco englobava de 30 a $150^{\circ}$ e o segundo arco englobava de 210 a $330^{\circ}$ ), com margens de $1,5 \mathrm{~cm}$ englobando próstata e terço proximal das vesículas seminais; as isodoses foram normalizadas (no isocentro) e foi escolhida a isodose de $95 \%$, que englobava o volume alvo.

Nas duas fases de tratamento a dose total de prescrição foi de 7.020 cGy. O centro do campo de tratamento foi colocado no centro da próstata, utilizando-se a tomografia de planejamento, porém, usando-se colimadores simétricos para abertura dos campos de tratamento; não foram utilizados blocos de colimação.

No planejamento conformado isocêntrico o esquema de tratamento foi simulado para o acelerador de $6 \mathrm{MeV}$, em duas fases. Para os pacientes de baixo risco, o GTV e o CTV era a próstata, o PTV consistiu na próstata e terço proximal das vesículas seminais mais margem de $1 \mathrm{~cm}$ em todas as direções, com exceção da margem posterior, onde a margem adicionada era de 0,8 $\mathrm{cm}$. Para pacientes de risco intermediário e alto, o GTV e o CTV1 (CTV da $1^{\mathrm{a}}$ fase de tratamento) eram a próstata e as vesículas seminais, o CTV2 (CTV da $2^{\text {a }}$ fase de tratamento) era a próstata e terço proximal das vesículas seminais. O PTV1 (PTV da $1^{\mathrm{a}}$ fase de tratamento) consistiu no CTV1 mais margem de $1 \mathrm{~cm}$ em todas as direções, com exceção da margem posterior, onde a margem adicionada era de $0,8 \mathrm{~cm}$. O PTV2 (PTV da $2^{\text {a }}$ fase de tratamento) consistiu no CTV2 mais margem de $1 \mathrm{~cm}$ em todas as direções, com exceção da margem posterior, onde a margem adicionada era de $0,8 \mathrm{~cm}$. Em todos os planejamentos era adicionada margem de bloco de $0,5 \mathrm{~cm}$.

$\mathrm{Na}$ primeira fase o arranjo de campos variou de quatro a seis, até dose de 5.040 cGy, isodose de prescrição escolhida englobando próstata mais vesículas seminais (em pacientes de médio e alto risco) e próstata e terço proximal das vesículas seminais (em pacientes de baixo risco). Na segunda fase o arranjo de campos variou de quatro a seis, até dose de 2.160 cGy, isodose de prescrição englobando próstata e terço proximal das vesículas seminais. Nas duas fases de tratamento a dose total foi de $7.200 \mathrm{cGy}$, prescrita na curva de isodose de $95 \%$ (normalização no isocentro).

\section{Métodos de avaliação}

Foram analisados os histogramas de dose-volume (HDV) para volumes alvos (CTV e PTV) e os órgãos bexiga, reto e coxofemoral dos planejamentos conformados realizados, comparando-os com os planejamentos não conformados. Os HDVs são apresentados na Figura 1.

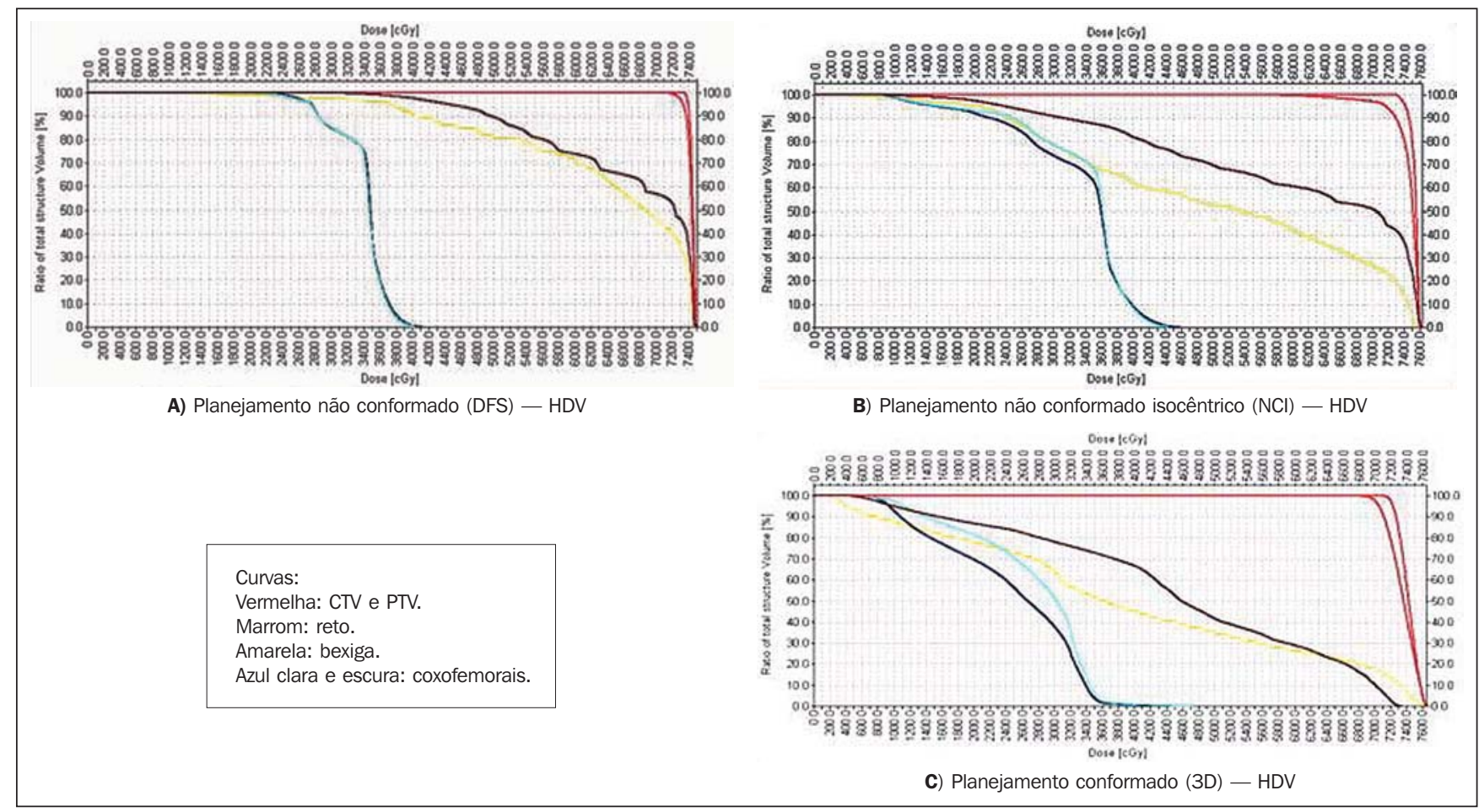

Figura 1. Comparação dos histogramas de dose-volume. 
Foram analisados, em ambos os planejamentos, as doses máximas, médias e medianas para o CTV e PTV.

Com relação aos órgãos de interesse analisados, no reto foram avaliadas as doses em $10 \%, 15 \%, 25 \%, 40 \%$ e $60 \%$ do seu volume (porcentagens analisadas na instituição), além das doses máximas, médias e medianas.

$\mathrm{Na}$ bexiga foram avaliadas as doses em $30 \%$ e $60 \%$ do seu volume (porcentagens analisadas na instituição), além das doses máximas, médias e medianas.

Nas articulações coxofemorais foram analisadas as doses máximas, médias e medianas em seu volume.

\section{Análise estatística}

Para análise dos resultados foi aplicada a análise de variância de Friedman. Fixou-se em 0,05 ou $5 \%(\alpha=0,05)$ o nível para a rejeição de hipótese de nulidade, assinalando-se com um asterisco os valores significantes e pela indicação NS os não significantes.

\section{RESULTADOS}

Os resultados obtidos com a análise dos planejamentos não conformados utilizando distância fonte-superfície fixa de $100 \mathrm{~cm}$, não conformados isocêntricos e conformados dos pacientes deste estudo serão apresentados a seguir.

As medianas das doses foram significativamente menores no planejamento conformado, ao se fazer a análise dos seguintes volumes no reto: $25 \%, 40 \%$ e $60 \%$ (Figura 2). As medianas das doses foram significativamente menores no planejamento conformado, ao se fazer a análise dos seguintes volumes na bexiga: $30 \%$ e 60\% (Figura 3).

Na comparação das doses máximas nas articulações coxofemorais, os planejamentos não conformados apresentaram doses medianas maiores; as medianas das doses médias e medianas nas articulações coxofemorais foram significativamente menores no planejamento conformado (Figuras 4 e 5).

Na comparação das doses máximas, médias e medianas no CTV e PTV, os planejamentos conformados obtiveram doses medianas maiores (Figuras 6 e 7).

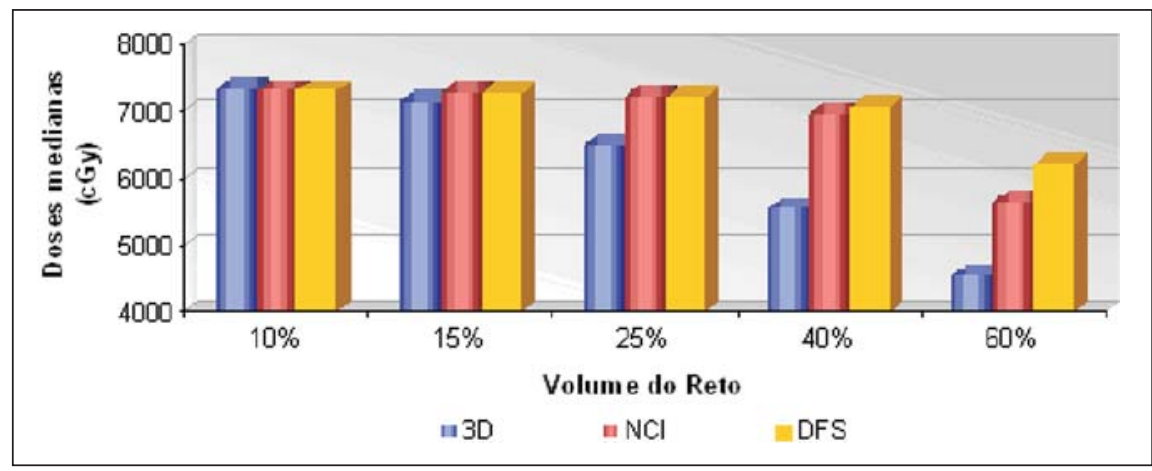

Figura 2. Comparação das doses em 10\%, 15\%, 25\%, 40\% e $60 \%$ do volume do reto.

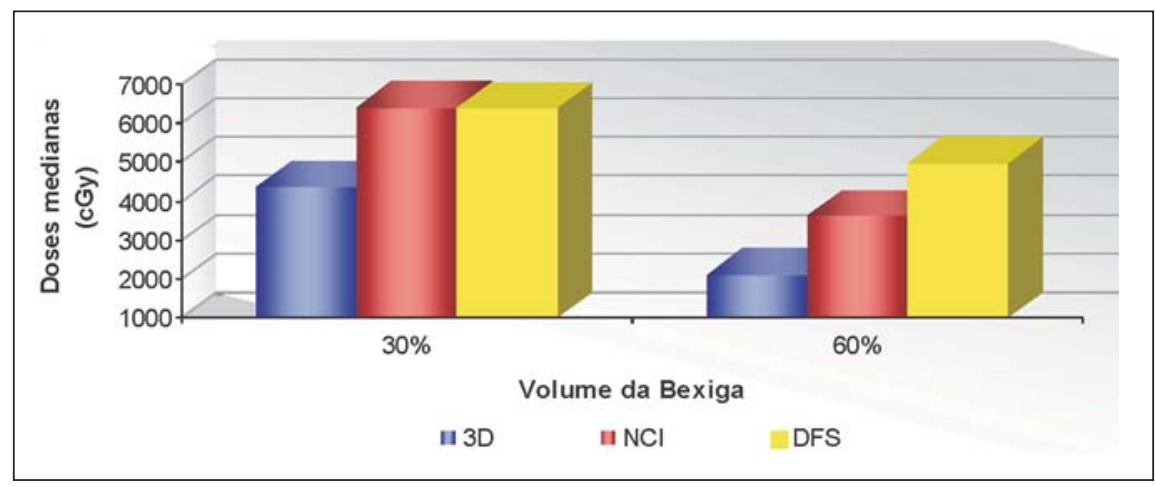

Figura 3. Comparação das doses em $30 \%$ e $60 \%$ do volume da bexiga.

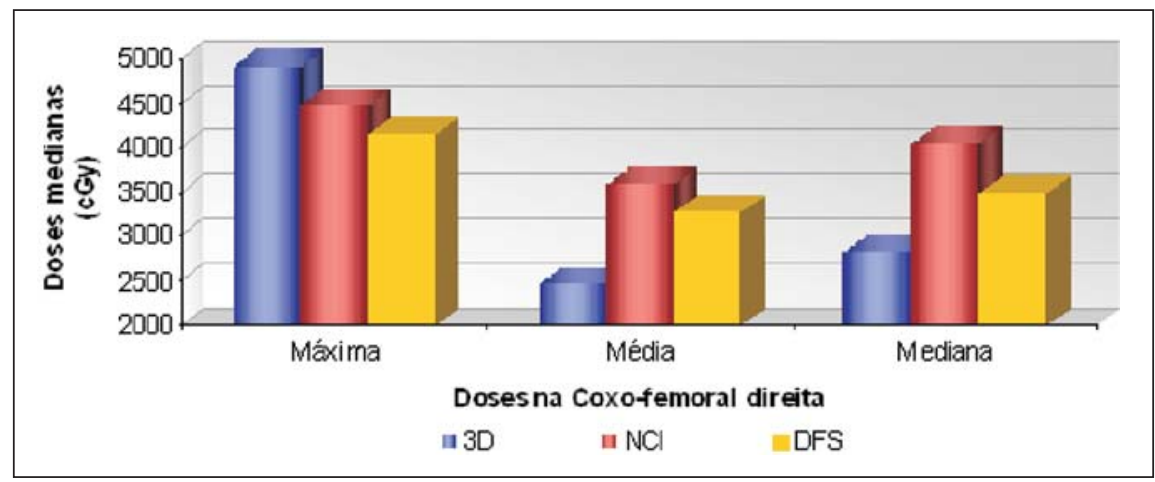

Figura 4. Comparação das doses na articulação coxofemoral direita.

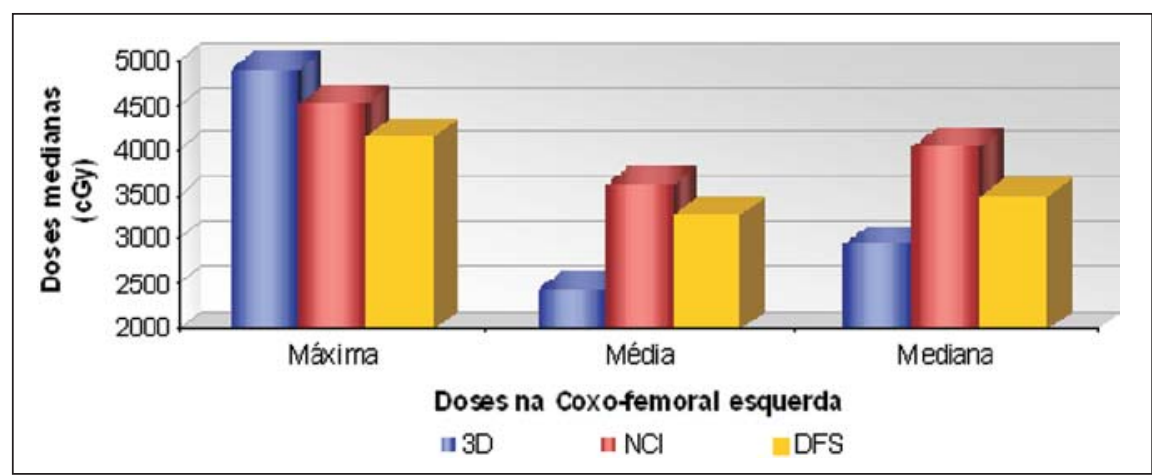

Figura 5. Comparação das doses na articulação coxofemoral esquerda. 


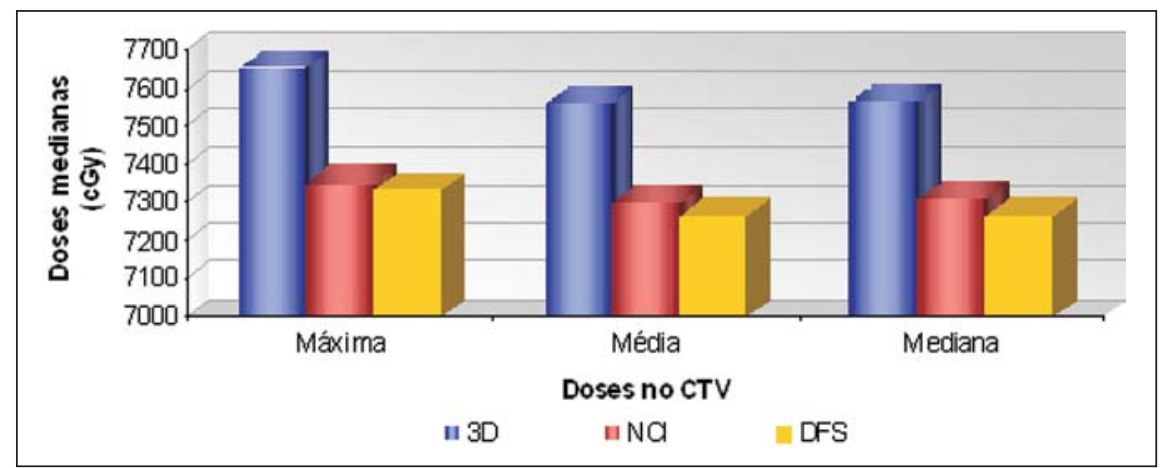

Figura 6. Comparação das doses no CTV.

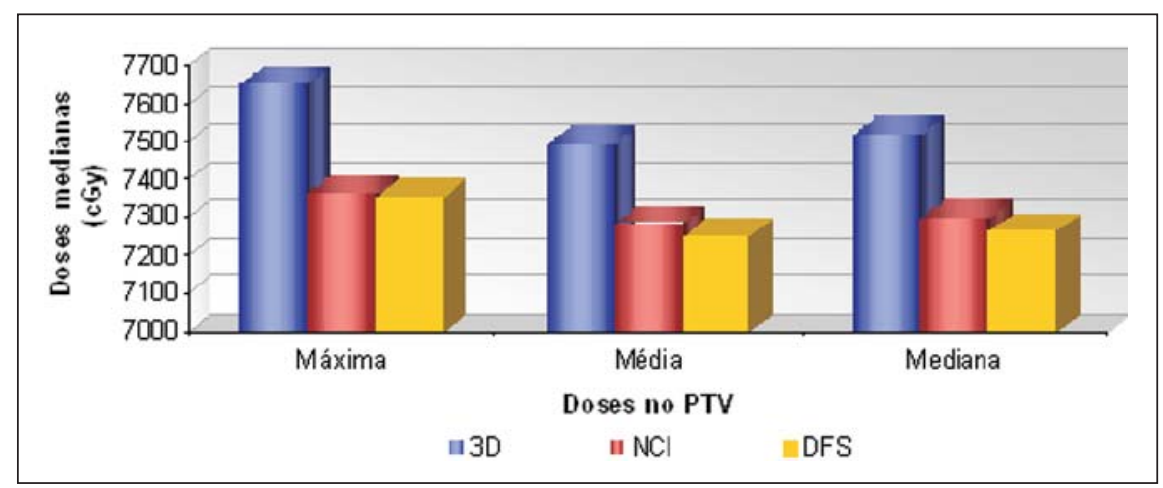

Figura 7. Comparação das doses no PTV.

\section{DISCUSSÃO}

Com relação aos volumes dos órgãos estudados, este trabalho encontrou os seguintes resultados: o volume mediano do reto foi de $63 \mathrm{~cm}^{3}$, o volume mediano da bexiga foi de $201 \mathrm{~cm}^{3}$, os volumes medianos das articulações coxofemorais direita e esquerda foram de $158,4 \mathrm{~cm}^{3}$ e $153 \mathrm{~cm}^{3}$, respectivamente, e o volume mediano do GTV foi de $51 \mathrm{~cm}^{3}$.

O estudo comparativo entre o planejamento convencional, o conformado e o de intensidade modulada relatou volume mediano de reto, bexiga, articulação coxofemoral e GTV de $33 \mathrm{~cm}^{3}, 79 \mathrm{~cm}^{3}, 111 \mathrm{~cm}^{3}$ e $76 \mathrm{~cm}^{3}$, respectivamente, nos planejamentos convencionais e conformados. Os volumes menores encontrados por Oh et al. podem ter sido decorrentes de os órgãos de risco só terem sido contornados $1,5 \mathrm{~cm}$ abaixo e acima da próstata e das vesículas seminais $^{(2)}$.

Sale et al., em trabalho comparativo de doses e volumes para câncer de próstata com técnicas convencional e conformada, encontraram GTV com volume mediano em $64,2 \mathrm{~cm}^{3}$, com os órgãos de risco tendo sido contornados $2,0 \mathrm{~cm}$ acima e abaixo da próstata. As vesículas seminais não foram incluídas no $\mathrm{GTV}^{(7)}$.

Nossos resultados apresentaram volumes maiores do que os da literatura, em razão das diferentes técnicas usadas nos contornos dos alvos e órgãos de risco.

As doses médias e medianas encontradas nos HDVs do reto e da bexiga no planejamento conformado foram significativamente menores, quando comparadas às doses encontradas no planejamento convencional (com exceção da dose média e mediana em $10 \%$ do volume do reto).

Perez et al. relataram dose média maior na bexiga e no reto com a técnica convencional, quando comparada à conformada, que usa feixes oblíquos para reduzir a radiação nessas estruturas ${ }^{(8)}$.

Oh et al. reportaram doses médias na bexiga de 2.132 a 2.711 cGy e doses médias no reto de 2.932 a 3.620 cGy no planejamento convencional. Já na técnica conformada, encontraram doses médias na bexiga de 1.241 a 1.952 cGy e doses médias no reto de 2.432 a 3.502 cGy, nos casos em que a dose de 4.500 cGy era prescrita no isocentro $^{(2)}$.

Outro trabalho comparativo (publicado em 2000 e atualizado em 2002) entre as técnicas estudadas demonstrou, na técnica conformada, porcentagem de 8,5 $\pm 11,8$ com dose $\geq 70$ Gy no reto, e na técnica convencional, porcentagem de $28,8 \pm 28,9$. $\mathrm{Na}$ bexiga, a porcentagem de volume com dose $\geq 70$ Gy foi de $6,3 \pm 8,4$ na técnica conformada e de 19,4 $\pm 24,4$ na técnica convencional $^{(\mathbf{8 , 9})}$. Nesses trabalhos de Perez et al., a técnica convencional foi realizada com dois arcos rotatórios de $120^{\circ}$ e margens de $2 \mathrm{~cm}$ da próstata. Já a técnica conformada foi realizada com sete campos, com colimações com cerobende ou colimador multifolhas $^{(\mathbf{8 , 9})}$.

Em estudo randomizado comparando toxicidade entre a radioterapia convencional e a conformada em câncer de próstata, Dearnaley et al. reportaram que o volume mediano de doses altas — definido como o volume de intersecção no espaço para os feixes de tratamento no planejamento conformado - foi reduzido em 39\% com o uso da técnica conformada. Esse estudo relatou que $48 \%$ menos de volume retal e $38 \%$ menos de volume vesical foram tratados na isodose de $90 \%$ no tratamento conformado, quando comparado com o tratamento convencional ${ }^{(\mathbf{1 0})}$.

Os dados obtidos no presente trabalho, referentes às doses máximas dos HDVs do reto e da bexiga, mostram que as doses máximas foram significativamente maiores no planejamento conformado. Acreditamos que tal fato se deveu à dose maior prescrita, que não foi compensada pelas colimações nem pelo maior número de entrada de campos.

Em estudo de Sale et al. sobre as duas técnicas de planejamento, o tratamento convencional utilizou, como volume alvo, área definida pela curva de isodose de $95 \%$ (englobando a próstata), e o tratamento conformado utilizou área definida pela próstata com margem de $1,5 \mathrm{~cm}$. Nos resultados não foram encontradas doses máximas significativamente maiores no volume de reto e bexiga no planejamento conformado. Entretanto, a dose em $100 \%$ do volume vesical e a dose em $100 \%, 90 \%$ e $50 \%$ do volume retal foi significativamente maior no planejamento conformado. Os 
autores argumentaram que isto ocorreu devido ao maior tamanho de campo no planejamento conformado, não compensado pelas colimações ${ }^{(7)}$.

Variações de doses máximas foram observadas no reto e na bexiga, respectivamente, de 4.807 a 4.855 cGy e 4.661 a 4.803 cGy no planejamento com técnica convencional, e de 5.072 a 5.304 cGy e 4.705 a 5.078 cGy no planejamento conformado, quando prescrita dose de 4.500 cGy no isocentro ${ }^{(2)}$.

Nossos resultados mostram que as doses máximas nas articulações coxofemorais foram significativamente maiores no planejamento conformado, enquanto as doses médias e medianas nas articulações coxofemorais foram significativamente menores nessa técnica.

Com relação às doses na articulação coxofemoral, a literatura não mostra nítida vantagem entre a técnica conformada e a convencional. Foram relatadas doses máximas e médias, respectivamente, de 2.718 a 2.891 cGy e de 1.614 a 2.537 cGy no planejamento convencional. Já no planejamento conformado, foram relatadas doses máximas e médias de 3.539 a 4.901 cGy e de 1.850 a 2.443 cGy, respectivamente ${ }^{(2)}$.

No presente trabalho, as doses máximas, médias e medianas encontradas nos HDVs do CTV e PTV foram significativamente maiores no planejamento conformado. Esses dados concordam com a literatura, que refere que a técnica conformada prescreve doses maiores no PTV, quando comparada à técnica convencional.

Segundo Oh et al., a dose média no PTV variou de 4.455 a 4.547 cGy com a técnica convencional, e de 4.671 a 4.896 cGy com a técnica conformada ${ }^{(2)}$. Doses em $100 \%$, $90 \%$ e $50 \%$ do GTV e PTV significativamente maiores no planejamento conformado, quando comparado ao planejamento convencional, também foram relatadas ${ }^{(7)}$.

Outros autores relataram doses média e máxima no tumor, respectivamente, de $69,8 \pm 2.6$ Gy e $71,7 \pm 2,4$ Gy na técnica conformada, e de $69,7 \pm 2,8$ Gy e $71,3 \pm$ 2,8 Gy na técnica convencional ${ }^{(8)}$.

Pollack et al. realizaram estudo comparativo entre dois grupos de pacientes: um grupo que recebeu dose de $68 \mathrm{~Gy}$, tratado com técnica convencional, e outro que recebeu dose de $78 \mathrm{~Gy}$, tratado na primeira fase com técnica convencional, com posterior reforço de dose com técnica conformada. Esse estudo mostrou que uma porcentagem significativamente maior da bexiga recebeu doses maiores que $60 \mathrm{~Gy}$ no grupo tratado exclusivamente com técnica convencional. Com relação à porcentagem de volume do reto tratada acima de $60 \mathrm{~Gy}$, não houve diferenças estatísticas entre os grupos. O volume da próstata e das vesículas seminais que recebeu mais que $95 \%$ da dose prescrita foi significativamente maior no grupo que recebeu reforço de dose com técnica conformada ${ }^{(11)}$.

Em estudo de análise entre a técnica convencional (quatro campos) e duas técnicas conformadas (quatro e seis campos), Magrini et al. verificaram que as doses médias no reto, nas técnicas conformadas (75\% da dose do isocentro), foram menores que na técnica convencional $(79 \%$ da dose do isocentro), embora não tenham alcançado significância estatística. Nesse estudo foi visto que a dose média na bexiga foi estatisticamente menor nas técnicas conformadas com quatro e seis campos (57\% e 50\% da dose do isocentro, respectivamente) do que na técnica convencional (79\% da dose do isocentro). A porcentagem do CTV e PTV que recebeu mais de $95 \%$ da dose do isocentro foi estatisticamente maior nas técnicas conformadas ${ }^{(\mathbf{1 2})}$.

Os resultados do presente estudo mostram o benefício da utilização do sistema de planejamento tridimensional no tratamento radioterápico do câncer de próstata. Nossos resultados demonstram que mesmo com dose maior de prescrição na técnica conformada é possível alcançar doses maiores nos volumes alvos, com doses menores nos órgãos de risco.

\section{CONCLUSÃO}

O presente estudo mostra que é possível entregar doses maiores no volume alvo e doses menores em órgãos críticos quando se utiliza planejamento conformado.

\section{REFERÊNCIAS}

1. Leibel SA, Fuks Z, Zelefsky MJ, et al. Technological advances in external-beam radiation therapy for the treatment of localized prostate cancer Semin Oncol. 2003;30:596-615.

2. Oh CE, Antes K, Darby M, et al. Comparison of 2D conventional, 3D conformal, and intensitymodulated treatment planning techniques for patients with prostate cancer with regard to targetdose homogeneity and dose to critical, uninvolved structures. Med Dosim. 1999;24:255-63.

3. Fletcher GH. Textbook of radiotherapy. 3rd ed. Philadelphia: Lea \& Febiger; 1980

4. Perez CA, Halperin EC, Brady LW, et al. Principles and practice of radiation oncology. 4th ed Philadelphia: Lippincott Williams \& Wilkins; 2004.

5. Brundage M, Lukka H, Crook J, et al. The use of conformal radiotherapy and the selection of radiation dose in $\mathrm{T} 1$ or T2 low or intermediate risk prostate cancer - a systematic review. Radiother Oncol. 2002;64:239-50.

6. Mangar SA, Huddart RA, Parker CC, et al. Technological advances in radiotherapy for the treatment of localised prostate cancer. Eur J Cancer. 2005;41:908-21.

7. Sale CA, Yeoh EE, Scutter S, et al. 2D versus 3D radiation therapy for prostate carcinoma: a direct comparison of dose volume parameters. Acta Oncol. 2005;44:348-54

8. Perez CA, Michalski JM, Purdy JA, et al. Threedimensional conformal therapy or standard irradiation in localized carcinoma of prostate: preliminary results of a nonrandomized comparison. Int J Radiat Oncol Biol Phys. 2000;47:629-37.

9. Perez CA, Michalski JM, Mansur D, et al. Threedimensional conformal therapy versus standard radiation therapy in localized carcinoma of prostate: an update. Clin Prostate Cancer. 2002;1:97104.

10. Dearnaley DP, Khoo VS, Norman AR, et al. Comparison of radiation side-effects of conformal and conventional radiotherapy in prostate cancer: a randomised trial. Lancet. 1999;353:267-72.

11. Pollack A, Zagars GK, Starkschall G, et al. Conventional vs. conformal radiotherapy for prostate cancer: preliminary results of dosimetry and acute toxicity. Int J Radiat Oncol Biol Phys. 1996;34: 555-64.

12. Magrini SM, Cellai E, Rossi F, et al. Comparison of the conventional 'box technique' with two different 'conformal' beam arrangements for prostate cancer treatment. Cancer Radiother. 1999;3: 215-20. 